\title{
The Effect of Electron Diffusion on the Instability of a Townsend Discharge
}

\author{
Vladimir Mikhailenko \\ Research Institute of Computer, Information and Communication, Pusan National University, Pusan 609-735 \\ (Received May 11, 2012, Revised May 18, 2012, Accepted May 18, 2012) \\ The role of the electron diffusion on the stability of a Townsend discharge was investigated \\ with the linear stability theory for the one-dimensional fluid equation with drift-diffusion \\ approximation. It was proved that the discovered instability occurs as a result of the coupled \\ action of electron diffusion and the perturbed electric field by space charge. The larger electron \\ diffusion results in the faster growth rate at the regime of small perturbation of the electric \\ field by space charges.
}

Keywords : Electron diffusion instability, Townsend discharge

\section{Introduction}

There has been considerable interest in non-thermal atmospheric pressure glow discharges over last time due to the increased variety of their industrial applications Fridman and Lee [1,2]. The basic feature of the non-thermal discharges is that majority of the energy of the applied electric field goes into electrons, instead of heating the entire gas in the discharge cell. Numerous experiments show that depending on the parameters of the discharge, atmospheric pressure glow discharge is realized in two forms: a Townsend and glow discharges. A Townsend discharge is the simplest type of glow discharges. It is characterized by the absence of quasi-neutral plasma--the absolute value of the ion density exceeds much that of the electron density. The applied electric field is weakly disturbed by spatial charge, and the discharge current is governed mainly by the processes of the electron emission from the cathode.
The current of a Townsend discharge is only limited by the external circuit. The transition to a glow discharge occurs when the space charge in a Townsend discharge becomes large enough to cause a significant disturbance of the applied field.

The physics of space charge driven transitions of a Townsend discharge to subnormal, normal, and further to abnormal glow has attracted considerable attention of the discharge research community Melekin, Phelps, Kolobov, Kaganovich, Koch, Bruhn, Amiranashvili, et al [3-9] Numerous experimental, analytical, and numerical investigations provide deep insight into amazing variety of spatio-temporal processes, which are responsible for such transitions. Usually, it is assumed that electron diffusion is ignorable effect in the operation of the Townsend discharge, and a possible instabilities are connected with space charge of the ionized species alone [10]. In this paper, we present the results of the investigations of the dc glow discharges in the framework

\footnotetext{
* [E-mail] vladimir@pusan.ac.kr
} 
of the linear stability theory with the eigenvalue solver of COMSOL Multiphysics software. The stability of a Townsend discharge for the regime investigated in this research is far from the previously reported transitions, as the space charge is too small to produce any significant distortion of the applied electric field. We found, however, that even in these conditions de discharge appears unstable. The derivation of the basic equations and boundary conditions for the dimensionless variables corresponding to the parameter regime of the Townsend discharge is presented in Section 2. In Section 3, we present the results of the numerical calculations of the stability properties of the Townsend discharge. Finally, summary and discussions are presented in Section 4 .

\section{П. Basic Equations and Boundary Conditions}

The simplest set of equations containing the basic physics necessary for the investigation of the glow discharge stability comprises the well-known continuity equations in the drift-diffusion approximation. The density of electrons, $N_{e}$, and that of positive ions, $N_{i}$, are coupled in Poisson's equation for the electrostatic potential $\Phi[5]$,

$$
\begin{aligned}
& \frac{\partial N_{i}}{\partial_{t}}-\frac{\partial}{\partial Z}\left(\mu_{i} N_{i} E\right)=N_{e} a(E) \mu_{e} E, \\
& \frac{\partial N_{e}}{\partial t}+\frac{\partial}{\partial Z}\left(-D_{e} \frac{a N_{e}}{\partial Z}+\mu_{e} N_{e} E\right)=N_{e} a(E) \mu_{e} E, \\
& \varepsilon_{0} \frac{\partial E}{\partial Z}=e\left(N_{e}-N_{i}\right),
\end{aligned}
$$

where $D_{e}, \mu_{i}$, and $\mu_{e}$ are the electron diffusion coefficient, the mobility of ions, and that of electrons, respectively; $\alpha(E)$ is Townsend's ionization coefficient [10] and $E$ is the electric field; $\varepsilon_{0}$ is the permittivity of free space, $e$ is the elementary charge, and $t$ is time. the $Z$-axis is directed from the cathode to the anode, and thus is opposite to the electric field. For the Townsend limit considered in this study, both the mobility and the electron diffusion coefficient, which otherwise depend on the local electric field, can be assumed to be constant. Because in the Townsend mode electric field is practically constant, local field approximation is applicable to ionization rate $\alpha$. Furthermore, the process of dissociative recombination and ion diffusion (we assume that electron temperature exceeds greatly the ion temperature) are completely neglected. In contrast, the axial diffusion of electrons is taken into account. The boundary conditions are taken in the Townsend form. At the cathode $(Z=0)$, the electron flux is generated by the secondary electron emission by ion flux such that

$$
-D_{e} \frac{\partial N_{e}}{\partial Z}+N_{e} \mu_{e} E=\gamma N_{i} \mu_{i} E
$$

where $\gamma$ is the secondary electron emission coefficient. At the anode $(Z=L)$,

$$
\frac{\partial N_{e}}{\partial Z}=0, N_{i}=0
$$

We assume that the discharge current is maintained unperturbed by the external circuit (the limiting case of a very high ballast or, equivalently, a current-controlled discharge). By definition, the discharge current density is evaluated at the anode and is given by [11]

$$
J=-e N_{e} \mu_{e} E+\varepsilon_{0} \frac{\partial E}{\partial t} .
$$

The boundary conditions at the wall of the discharge vessel are not relevant for the present onedimensional study.

We are interested in processes on the time scale longer than the ion transit time

$$
t_{0}=\frac{L}{\mu_{i} E}
$$

and therefore the time derivative of ion density is 
considered as a very small perturbation. On this time scale, the time dependency of electron density is eliminated adiabatically and the time derivative of electron density in Eq. (2) is set to zero. It is convenient to introduce the dimensionless times $\tau$, length $\mathrm{z}$, and electric field $\hat{E}$ such that

$$
\tau=\frac{t}{t_{0}}, z=\frac{Z}{L}, \hat{\mathrm{E}}=\frac{E}{E_{0}}
$$

A significant parameter $\varepsilon_{e}$ is introduced as

$$
\varepsilon_{e}=\frac{D_{e}}{\mu_{e} E_{0} L}=\frac{T_{e}}{e L E_{0}}
$$

which determines the relative value of the diffusive term to the drift term in Eq. (2). By assuming that dimensionless anode current is equal to unity, we obtain the following dimensionless electron density $n_{e}$ from boundary condition [Eq. (6)],

$$
n_{e}=\frac{N_{e} e \mu_{e} E_{0}}{J} .
$$

Similarly,

$$
n_{i}=\frac{N_{i} e \mu_{i} E_{0}}{J} .
$$

we use for the dimensionless ion density $n_{i}$. In dimensionless form, the governing equations $(1) \sim(3)$ are modified as

$$
\begin{aligned}
& \frac{\partial n_{i}}{\partial \tau}-\frac{\partial}{\partial z}\left(n_{1} E\right)=\hat{\mathrm{a}} n_{e}|E|, \\
& -\varepsilon_{e} \frac{\partial^{2} n_{e}}{\partial z^{2}}+\frac{\partial}{\partial z}\left(n_{e} E\right)=\hat{\mathrm{a}} n_{e}|E|, \\
& \frac{\partial \hat{\mathrm{E}}}{\partial z}=\delta\left(\frac{\mu_{i}}{\mu_{e}} n_{e}-n_{i}\right) \approx-\delta n_{i},
\end{aligned}
$$

where $\hat{\mathrm{a}}=\alpha \mathrm{L}$ and the parameter $\delta$,

$$
\delta=\frac{J L}{\varepsilon_{0} \mu_{i} E_{0}^{2}},
$$

determines the distortion of the external ambient electric field by space charge [12] from Eq. (14). Also, $\varepsilon_{e}$ is assumed to be small for a Townsend discharge. To facilitate the simplifications which were made in the calculations, we present typical values of the used parameters for a cold atmospheric Townsend discharge: $J=0.1 \mathrm{~mA} / \mathrm{m}^{2}, E_{0}=3.5 \times 10^{5} \mathrm{~V} / \mathrm{m}, L=0.5 \mathrm{~mm}$, $\alpha=7,000 \mathrm{~m}^{-1}, \mu_{e}=10.6 \mathrm{~m}^{2} \mathrm{~V}^{-1} \mathrm{~s}^{-1}, \mu_{i}=2.2 \times 10^{-3} \mathrm{~m}^{2} \mathrm{~V}^{-1}$ $\mathrm{s}^{-1}, D_{e}=1 \mathrm{~m}^{2} / \mathrm{s}, \gamma=0.03, T_{e}=1 \mathrm{eV}$, and $T_{i}=0.026 \mathrm{eV}$. With these parameters, $\mathrm{a}=3.5, t_{0}=1.2 \times 10^{-6} \mathrm{~s}, \delta=2 \times$ $10^{-6} \mathrm{~s}, \delta=2 \times 10^{-3}$, and $\varepsilon_{e}=8 \times 10^{-3}$.

\section{Numerical Analysis}

The linear stability analysis of the Townsend discharge treats the density of electrons and ions in a conventional form of

$$
\begin{aligned}
& n_{e}\left(z, \tau ; \varepsilon_{e}, \delta\right)=n_{e 0}\left(z ; \varepsilon_{e}, \delta\right)+n_{e 1}\left(z ; \varepsilon_{e}, \delta\right) e^{\lambda \tau} \\
& n_{i}\left(z, \tau ; \varepsilon_{e}, \delta\right)=n_{i 0}\left(z ; \varepsilon_{e}, \delta\right)+n_{i 1}\left(z ; \varepsilon_{e}, \delta\right) e^{\lambda \tau}
\end{aligned}
$$

The numerical analysis of this system was executed with COMSOL code with a goal to estimate the relative effect of the different terms in these systems on the magnitude and sign of the stability parameter $\lambda$. For this goal we consider the set of the reduced equations and calculate parameter $\lambda$. We have analyzed the following reduced systems:

- Systems without electron density in Poisson's equation.

- In addition to 1 , the density of ions in Poisson's equation is neglected for the steady-state, i.e. $n_{i o}=0$.

- In addition to case 2 , the electron diffusion $D_{e}$ in the boundary condition is neglected for the steady state solution $n_{e o}$ [case (b) in the Fig. 1].

- In addition to case 3 , the electron diffusion $D_{e}$ is neglected [case (c) in the Fig. 1].

Fig. 1 shows the real parts of the dimensionless 
stability parameter $\hat{\lambda}=\lambda L / \mu_{i} E$ with the variation of $\delta$. Here, case (a) means the case with all terms in Eqs. (12) (14). It was observed that cases $1 \sim 3$ show almost the same tendency as those with full equations. However, case 4 shows only stable solutions with negative $\lambda$ as shown in case (c) of Fig. 1. The discovered instability appears as the result of the coupled interaction of the electron diffusion and the electric field by space charge, determined by the parameter $\varepsilon_{e}$ and $\delta$, respectively. The instability disappears, when electron diffusion or effects of electric field of the ionized species (parameter $\delta$ ) are ignored.

Fig. 2 show the dependency of the imaginary part of $\hat{\lambda}$ for the variation of $\delta$ for a fixed $\varepsilon_{e}=0.008$, which means oscillation frequency. The effect of is negligible for the oscillation frequency. Another interesting result is that the bifurcation point for $\varepsilon_{e}$ exists, which depends on the parameter $\delta$ that distinguishes two regimes of the instability: exponential growth with $\operatorname{Im} \lambda=0$ (for small values of $\delta<1.5 \times 10^{-3}$ ), and oscillatory regime with $\operatorname{Im} \lambda<0$ for greater $\delta$ values.

Fig. 3 shows that the stability of the discharge depends not only on the magnitude of the parameter $\delta$, but from the parameter $\varepsilon_{e}$ also, which was neglected in previous study [3]. For the growth rate $\hat{\lambda}$, there is a threshold value of the parameter $\varepsilon_{e}$, below which instability does not exist (i.e. negative or zero real values of $\lambda$ ). Therefore, electron diffusion is an essential process in the development of the instability of the Townsend discharge.

\section{Conclusion}

In this work, by solving the eigenvalue problem for the stability parameter $\lambda$, we have shown that a Townsend discharge is unstable due to joint action of the ionization process and electron diffusion. Accounting for this, the discovered instability is

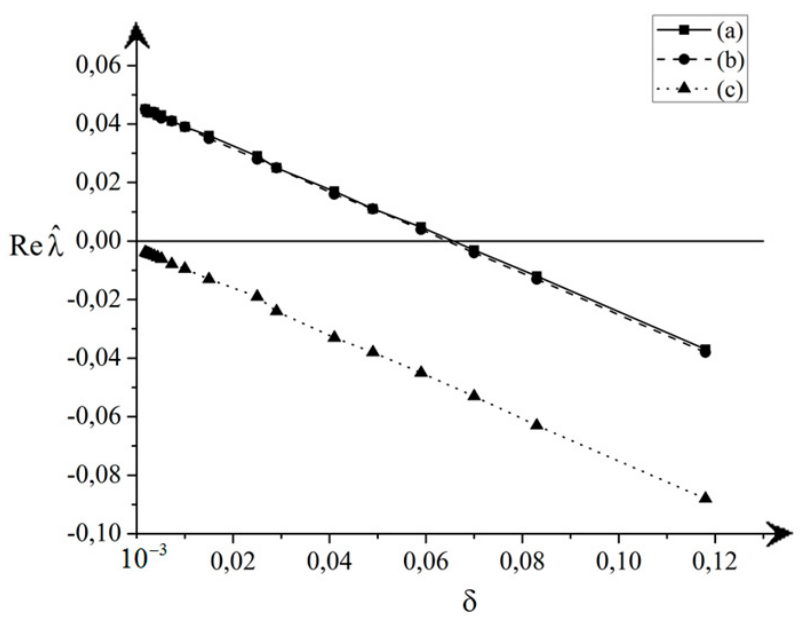

Figure 1. The dependence of the real part of $\hat{\lambda}$ on $\delta$ for $\varepsilon_{e}=8 \cdot 10^{-3}$.

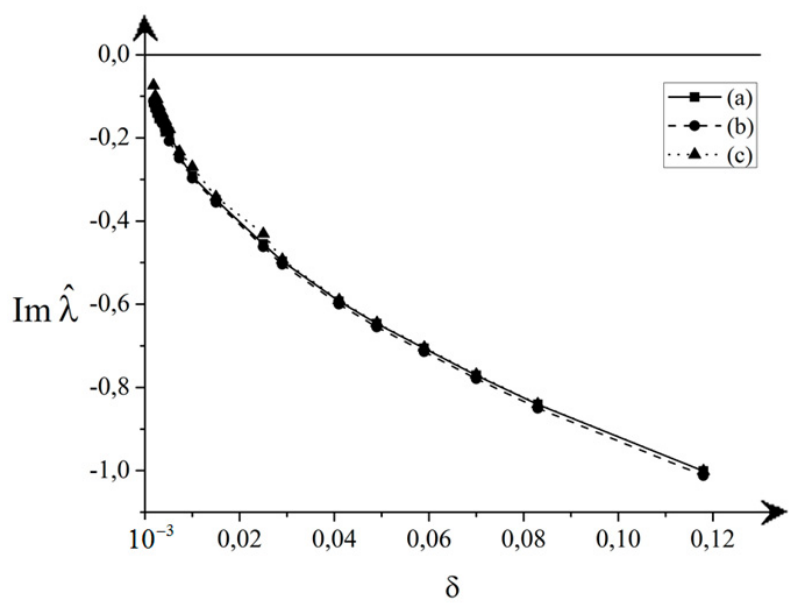

Figure 2. The dependence of the imaginary part of $\hat{\lambda}$ on $\delta$ for $\varepsilon_{e}=8 \cdot 10^{-3}$.

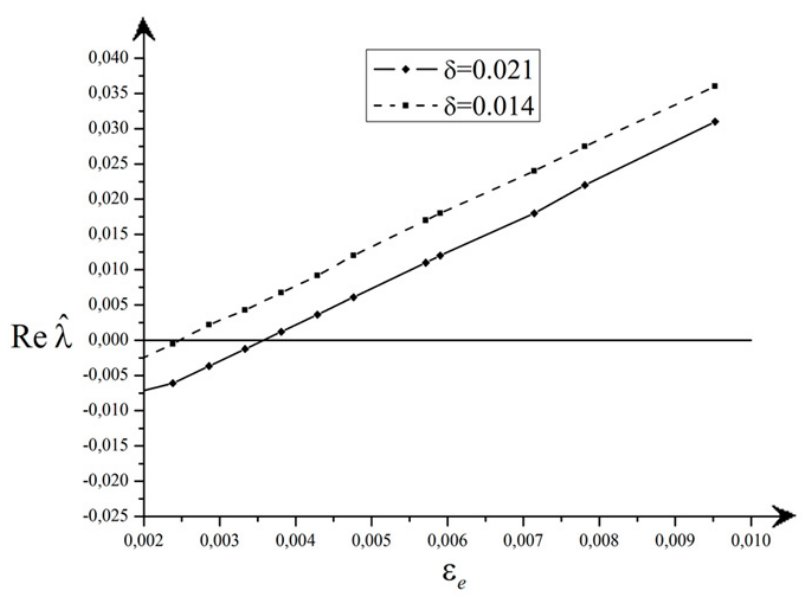

Figure 3. The dependence of the real part of $\hat{\lambda}$ on $\varepsilon_{e}$ for $\delta=0.021$ and $\delta=0.014$. 
identified as a diffusive instability.

\section{Acknowledgments}

This research was supported by National R\&D Program through the National Research Foundation of Korea (NRF) funded by the Ministry of Education, Science and Technology (Grant N 2011-0020631).

\section{References}

[1] A. Fridman, A. Chirokov, and A. Gutsol, J. Phys. D. Appl. Phys. 38, R1 (2005).

[2] H. W. Lee, G. Y. Park, Y. S. Seo, Y. H. Im, S. B. Shim, and H. J. Lee, J. Phys. D. Appl. Phys. 44, 053001 (2011).

[3] V. N. Melekin and N. Yu, Naumov. Sov. Phys.
Tech. Phys. 29, 888 (1984).

[4] A. V. Phelps, Z. L. Petrovic, and B. M. Jelenkovic, Phys. Rev. E 47, 2825 (1993).

[5] V. I. Kolobov and A. Fiala, Phys. Review E 50, 3018 (1994).

[6] I. D. Kaganovich, M. A. Fedotov, and L. D. Tsendin, Tech. Phys. 39, 241 (1994).

[7] B. P. Koch, N. Goepp, and B. Bruhn, Phys. Review E 56, 2118 (1997).

[8] B. Bruhn, A. Richter, and B. May, Phys. Plasmas 15, 053505 (2008).

[9] S. Amiranashvili, S. Gurevich, and H. G. Purvins, Phys. Review E 71, 066404 (2005).

[10] Yu. P. Raizer, Gas Discharge Physics (Springer, Berlin, 1991), pp.167-244.

[11] P. G. C. Almeida, M. S. Benilov, and M. J. Faria, J. Phys. D. Appl. Phys. 44, 415203 (2011).

[12] M. S. Benilov, Phys. Review E 77, 036408 (2008). 


\title{
전자 확산 효과가 Townsend 방전 불안정성에 미치는 영향
}

\author{
블라디미르 미카일렌코
}

컴퓨터 정보통신 연구소, 부산대학교, 부산 609-735

(2012년 5월 11일 받음, 2012년 5월 18일 수정, 2012년 5월 18일 확정)

\begin{abstract}
드리프트-확산 근사식을 이용한 1차원 유체 방정식으로부터 선형적 안정성 이론을 전개하여 Tosend 방전에서 전자 확산이 불 안정성에 미치는 영향을 관찰하였다. 본 연구에서 관찰된 바에 따르면 Townsend 불안정성은 전자 확산과 공간 전하에 의해 형성된 전기장의 효과가 결합되어 발생하며, 공간전하에 의한 효과가 작은 영역, 즉 방전 전류가 낮은 영역에서는 전자 확산 효과가 커질수록 불안정이 더 빨리 진행된다는 것이 발견되었다.
\end{abstract}

주제어: 전자 확산 불안정성, 타운젠트 방전

*[전자우편] vladimir@pusan.ac.kr 